Evaluar.

2003, $n^{\circ} 3$ (julio)
Laboratorio de Evaluación Psicológica y Educativa. Facultad de Psicología Universidad Nacional de Córdoba (Argentina). ISSN N ${ }^{\circ} 1667-4545$

\title{
Inventario de Autoeficacia para Inteligencias Múltiples: Fundamentos Teóricos y Estudios Psicométricos.
}

\author{
Pérez, E. *, Beltramino, C.*, Cupani, M.* \\ *Lab. de Evaluación Psicológica y Evaluativa. Facultad de Psicología. Universidad Nacional de Córdoba
}

\begin{abstract}
Resumen: El propósito de este trabajo es describir la construcción y validación de un inventario que evalúa la autoeficacia que los individuos poseen en varias características asociadas con las inteligencias múltiples propuestas por Gardner y con fines de Orientación Vocacional. Otro objetivo es revisar los descubrimientos recientes respecto a los fundamentos neuropsicológicos de las inteligencias múltiples. Este instrumento se integra en un Sistema de Orientación Vocacional asistido por computadora, el SOVI 3 (Fogliatto \& Pérez, en prensa), como módulo de exploración psicológica junto al Cuestionario de Intereses Profesionales-Revisado (Fogliatto, Pérez, Olaz \& Parodi, 2003).
\end{abstract}

Palabras Clave: Autoeficacia-Inteligencias Múltiples- Orientación Vocacional

\section{Introducción}

En nuestro país hay un considerable déficit en medidas locales de evaluación del comportamiento vocacional. Los únicos instrumentos diseñados en la región son un Cuestionario de Intereses Profesionales (Fogliatto, 1991) y un Sistema de Orientación para Carreras Informatizado (Fogliatto, Pérez, 1997; 2001). No se poseen medidas de la autoeficacia (Bandura, 1997) ni de las Inteligencias Múltiples (Gardner, 1994) que permitan su empleo en Orientación Vocacional.

La Teoría de las Inteligencias Múltiples (Gardner, 1994; 1999) es un modelo alternativo a la concepción unitaria de la inteligencia que propone un conjunto de potenciales biopsicológicos para analizar información que pueden ser activados en un marco cultural y que les permiten resolver problemas o crear productos valiosos en su medio cultural. Este enfoque teórico tiene numerosas implicancias educacionales. En el terreno de la Orientación Vocacional, específicamente, se lo ha propuesto como modelo de trabajo para los orientadores, quienes podrían considerar los patrones individuales en múltiples inteligencias para asesorar a sus clientes en sus planes de carrera (Shearer, 
1999). Con este fin este autor diseñó la Multiple Intelligences Developmental Assessment Scales, una medida de autoinforme de las inteligencias múltiples con estudios psicométricos rigurosos. (Shearer, 1996).

El constructo de la autoeficacia ha revelado un considerable valor heurístico en el campo de la investigación vocacional. Bandura (1987) ha definido autoeficacia como "creencias de las personas acerca de sus capacidades que les permiten organizar y ejecutar cursos de acción requeridos para alcanzar determinados tipos de rendimiento". Este concepto fue introducido en la literatura vocacional por Hackett \& Betz (1981) y se ha demostrado su valor predictivo con relación a variables significativas del comportamiento vocacional, tales como rendimiento académico y elección de carreras (Hackett y Lent, 1992; Multon, Brown \& Lent, 1991). Como resultado de esta utilidad predictiva, el constructo de la autoeficacia se ha convertido en uno de los mas prominentes en la literatura vocacional.

Los investigadores social-cognitivos han demostrado que la confianza que poseen los estudiantes en las habilidades requeridas por un área académica es frecuentemente mejor predictor del éxito posterior en esa área que sus habilidades objetivas (Bandura, 1997; Pajares et al., 2001). En parte, esto es debido al hecho que tales autopercepciones ayudan a los individuos a determinar que hacer con los conocimientos y habilidades que poseen. Por estas razones, desde la teoría social cognitiva, se hipotetiza que la confianza que los estudiantes poseen en resolver problemas relacionados con las inteligencias múltiples de Gardner (1994) podría ser mejor predictor de sus intenciones para emprender carreras en las cuales estas inteligencias sean requeridas, que su actual posesión de tales capacidades. Hasta el presente, sin embargo, no existen instrumentos que permitan evaluar tal confianza en las actividades relacionadas con las múltiples inteligencias.

El propósito de este trabajo es describir la construcción y validación de un instrumento que evalúa la autoeficacia que los individuos poseen en varias características asociadas con las inteligencias múltiples propuestas por Gardner y con fines de Orientación Vocacional. Otro objetivo es revisar los descubrimientos recientes respecto a los fundamentos neuropsicológicos de las inteligencias múltiples. 


\section{La Teoría de las Inteligencias Múltiples y sus Fundamentos Neurofisiológicos y Psicofisiológicos.}

Gardner (1999) examina cada posible inteligencia con un conjunto de criterios provenientes de las ciencias biológicas, el análisis lógico, la investigación psicológica y la psicología del desarrollo. De este modo, solo 8 habilidades merecen el nombre de "inteligencia" debido a que satisfacen la mayoría de estos criterios. A continuación, se describe brevemente cada inteligencia y se comentan algunos de los descubrimientos más recientes en relación a su fundamentos neurofisiológicos y psicofisiológicos.

La inteligencia Lingüística se relaciona con las habilidades para el lenguaje hablado y escrito, el aprendizaje de idiomas y el empleo de la lengua para alcanzar ciertos fines. La habilidad de procesar los mensajes lingüísticos con rapidez parece depender del lóbulo temporal izquierdo. Por este motivo, las lesiones en esta región neural o su desarrollo anormal casi siempre son suficientes para provocar dificultades en el lenguaje. La función interpretativa general y, específicamente, de los aspectos semánticos del lenguaje se encuentra ubicada en el lóbulo temporal izquierdo, detrás de la corteza auditiva primaria (es el plano temporal izquierdo, que es asimétrico, con predominio franco en el lóbulo izquierdo en la mayoría de las personas). Es la zona cortical donde se produce la comprensión del lenguaje, la denominada área de Wernicke. La segunda zona de integración interpretativa es el giro angular izquierdo que recibe la información visual y la integra en el área de Wernicke y su alteración provoca alexia y agrafia, así como la lesión de las conexiones que provienen desde la corteza visual al giro angular. Una tercera región de función específica en el lenguaje es el área de Broca, situada en una pequeña zona de la corteza frontal izquierda. Esta región almacena y controla los programas de articulación y ordenamiento silábico. Cuando estos programas se activan, a través del facículo arqueado (haz de fibras que trae lo interpretado desde el área de Wernicke) envía impulsos a la parte inferior de la corteza frontal ascendente que controla la fonación y emisión de los conceptos. Por tanto, las lesiones en el área de Wernicke producen una afasia de recepción, mientras la lesión del área de Broca produce una afasia de expresión (Ver Apéndice, Figura 2 y 3). Estas estructuras que forman el circuito básico que controla la entrada, elaboración, 
interpretación y salida del lenguaje se reúnen en un modelo denominado de WernickeGeschwind. (Geschwind, 1979; Gazzaniga, 1998).

La inteligencia Lógico-Matemática comprende capacidades para el análisis lógico de problemas, el cálculo numérico y la investigación científica. La habilidad para comprender relaciones y conceptos numéricos parece depender del hemisferio derecho y leer y producir signos matemáticos es más frecuentemente una función del izquierdo (Gardner, 1975). Existe un consenso frágil de que determinadas áreas cerebrales poseen especial importancia en cuestiones de lógica y matemática, los lóbulos parietales izquierdos y las áreas temporales y occipitales contiguas (Ver Apéndice, Figura 1). Luria (1966) concluye, que las lesiones en esta región cerebral pueden afectar también las capacidades de orientación en el espacio y de comprensión de algunas estructuras gramaticales como es el caso de las construcciones en voz pasiva. No obstante, estas áreas neurales implicadas no parecen ser tan indispensables para el pensamiento lógico y matemático como determinadas áreas lo son para el lenguaje, por ejemplo. Estudios electrofisiológicos (Gervais, 1982) demuestran que ambos hemisferios participan en la resolución de problemas matemáticos. Los estudios neurobiológicos modernos han permitido la localización de funciones en forma lateralizada, es decir con predominio en un hemisferio sobre el otro. El caso de las matemáticas es muy particular porque mientras el hemisferio izquierdo posee las cualidades para el desarrollo de la habilidad aritmética, el derecho es predominantemente espacial y, por consiguiente, controla la habilidad geométrica (Ameisen, 1996). Las habilidades lógico-matemáticas no se deterioran como resultado de daño cerebral focalizado (como en las inteligencias lingüística y musical) sino como resultado de enfermedades deteriorantes mas generales (como la demencia, por ejemplo).

La Inteligencia Musical comprende las habilidades para la ejecución, composición y apreciación de patrones musicales. La mayoría de las habilidades musicales están localizadas en el hemisferio derecho en los individuos normales. La inteligencia musical depende fundamentalmente de la percepción auditiva. Esta vía sensorial es de proyección predominantemente contralateral; el 75\% de sus neuronas proyectan sus axones al hemisferio opuesto y esto determina un predominio contralateral de la elaboración de lo oído. De ahí que el hemisferio derecho que recibe el mayor porcentaje de la audición izquierda sea el más capaz para la interpretación, 
elaboración y coordinación de las aferencias auditivas relacionadas con la música. Se ha comprobado que determinadas lesiones en los lóbulos frontal y temporal derecho causan pronunciadas dificultades para distinguir tonos y reproducirlos correctamente. Sin embargo, cuando las heridas se producen en regiones homólogas del hemisferio izquierdo, las habilidades musicales no resultan por lo común afectadas. También parece ser que la habilidad central de apreciación de la música es afectada por enfermedades del hemisferio derecho como la amusia (Aube, 1980). Los individuos afásicos presentan menores habilidades musicales; pero el hallazgo principal de estos estudios es que se puede padecer de afasia sin que se perciban impedimentos musicales significativos y que se puede quedar impedido musicalmente pero conservando, al mismo tiempo, las habilidades lingüísticas esenciales

La Inteligencia Cinéstésica-Corporal se relaciona con destrezas para usar el propio cuerpo o partes del mismo en la resolución de problemas o la creación de productos. La mayoría de las partes del cuerpo participan de una u otra manera en la ejecución de acciones motoras. Dentro del sistema nervioso, grandes porciones de la corteza, junto al tálamo, los ganglios basales y el cerebelo proporcionan información a la médula, estación intermedia en la ejecución de la acción (Ver Apéndice, Figura 1). La operación del sistema motor es extremadamente compleja y requiere la coordinación de una enorme variedad de componentes neurales y musculares en una forma diferenciada e integrada. La tendencia al predominio del hemisferio izquierdo en la actividad motora parece ser una predisposición de los seres humanos, al menos parcialmente bajo control genético (Gardner, 1994; Kandel, 1997). Las mitades izquierdas del cerebro son dominantes para la actividad motora. Las lesiones en las zonas del hemisferio izquierdo que dominan estas habilidades pueden producir impedimentos selectivos (Roy, 1982). Los neurólogos hablan de apraxias, conjunto de enfermedades relacionadas, en las que los individuos que en lo físico son capaces de realizar secuencias motoras y que pueden comprender un pedido de hacerlo, no pueden realizarlas en el orden o la forma apropiada. Si bien a menudo estas apraxias se presentan en combinación con afasias, existen muchas evidencias de que la apraxia no es sólo un desorden lingüístico o simbólico. Personas con graves problemas de comprensión de mensajes verbales muestran sorprendente conservación de la habilidad para ejecutar determinadas clases

Evaluar.

2003, $n^{\circ} 3$ (julio) 
de órdenes (movimiento del torso, por ejemplo); algunos estudios evidencian que el grado de deterioro para comprender diversos símbolos no está muy correlacionado con la habilidad para realizar acciones motoras voluntarias (Gardner, 1994). Otros investigadores han demostrado que los individuos que han perdido por completo su memoria verbal conservan, empero, la capacidad para aprender y recordar secuencias motoras y patrones de comportamiento complejos. También se han documentado casos de pacientes, normales en otros aspectos, que no pueden realizar ninguna acción motora voluntaria; son los apráxicos aislados, que muestran en forma esencialmente pura, la ausencia de inteligencia Cinestésico-Corporal (Gardner, 1994).

Por Inteligencia Espacial se entienden las aptitudes para reconocer y manipular patrones espaciales amplios o específicos. El hemisferio derecho del cerebro y, en particular, las porciones posteriores del mismo son las regiones más importantes para el procesamiento espacial. Si bien el daño de las regiones posteriores izquierdas pueden ocasionar graves déficits en la habilidad espacial, las capacidades de desenvolverse en un sitio, de reconocer objetos, caras y escenas y de observar detalles precisos están más estrechamente ligadas con las regiones posteriores derechas (Wasserstein, 1982). Se ha comprobado que las lesiones en las regiones parietales derechas provocan dificultades en la atención visual, representación y orientación espacial, producción de imaginería y memoria visual (Gardner, 1975). La dificultad en el procesamiento espacial es directamente proporcional a la magnitud de la lesión. La combinación de una lesión en el hemisferio izquierdo, aunque sea pequeña, con daño en el hemisferio derecho, basta para perjudicar seriamente el funcionamiento espacial de un individuo (Ver Apéndice, Figura 1).

La Inteligencia Interpersonal se vincula con habilidades para comprender la personalidad de otros seres humanos y trabajar efectivamente con ellos. La Inteligencia Intrapersonal es la capacidad de comprensión de los propios motivos, sentimientos y capacidades. Toda la investigación neuropsicológica coincide en señalar que los lóbulos frontales son las estructuras responsables de las inteligencias personales. Los déficits en esta región cerebral pueden interferir en el desarrollo y provocar formas patológicas de conocimiento inter e intrapersonal (Gardner, 1975). La destrucción de los lóbulos frontales en el adulto tienen efectos menores en la capacidad para resolver problemas lógicos pero pueden producir profundas alteraciones en la personalidad. Blumer (1975) 
ha demostrado que el daño en el área orbital de los lóbulos frontales provocan hiperactividad y euforia, mientras que las lesiones en la convexidad del lóbulo producen, generalmente, síntomas depresivos. Para Nauta (1981) esto es así debido a que estas estructuras son el sitio por excelencia de integración de los dos grandes ámbitos funcionales del cerebro: las regiones posteriores, ligadas al procesamiento de los datos sensoriales y los sistemas límbicos, donde se alojan las funciones emocionales y motivacionales del ser humano. Se necesita más evidencia concluyente acerca de las estructuras cerebrales que sustentan de manera diferencial a las dos inteligencias personales. Bear (1981) ha sugerido ideas interesantes al respecto trabajando con pacientes epilépticos del lóbulo temporal. El daño en las regiones dorsales de la corteza produce pérdida del sentido de atención hacia uno mismo e indiferencia, en estos pacientes. Por el contrario, las lesiones ventrales, provocan falta de interés por los estímulos externos y, como consecuencia, emisión inapropiada de respuestas sexuales o agresivas hacia otras personas.

Por último, la Inteligencia Naturalista, se caracteriza por competencias para el reconocimiento y clasificación de objetos del mundo natural (especies animales, por ejemplo) en su ambiente. Los estructuras neurales comprometidas en esta inteligencia no han sido bien establecidos aún. El reconocimiento de especies puede ser representado por diferentes vías en diferentes personas dependiendo, por ejemplo, de si las especies son reconocidas primariamente a través de dibujos o fotos o mediante interacciones directas con las plantas o animales. Debido a que la capacidad naturalista de los seres humanos está muy ligada a la de otros animales, debería ser posible confirmar cuales regiones cerebrales son decisivas para la percepción naturalista. La identificación de redes neurales comprometidas en formas específicas de reconocimiento, tales como la de rostros, puede suministrar importantes indicios para esta finalidad (Gardner, 1999).

\section{Método}

\section{Participantes}

Todos los sujetos de investigación fueron adolescentes que cursaban los dos últimos años de educación media del sistema educativo en Córdoba, Argentina. Sus edades

Evaluar.

2003, $n^{\circ} 3$ (julio) 
estuvieron comprendidas entre 16 y 20 años y se procuró que en cada muestra de investigación estuvieran proporcionalmente representadas las diferentes orientaciones educativas de los establecimientos de nivel medio de Argentina (Arte y Diseño, Música, Ciencias Naturales, Producción de Bienes y Servicios, Economía y Gestión de las Organizaciones, Ciencias Sociales). En cada estudio psicométrico específico se menciona, a continuación, el número de sujetos, la media de edad y el porcentaje por sexo.

\section{Instrumentos}

En todas las fases de la investigación se utilizó el Inventario de Autoeficacia para Inteligencias Múltiples, una prueba compuesta por ocho escalas, cada una representando una de las inteligencias propuestas por Gardner, elaboradas con fines de Orientación Vocacional para adolescentes que aspiran a ingresar a la Educación Superior.

El Inventario incluye 69 ítems que incluyen actividades académicas ("resolver problemas matemáticos", por ejemplo) y se solicita al examinado que evalúe en una escala de 1 a 10 la confianza que posee en su habilidad para desempeñarse en cada una de las tareas descriptas por los ítems (Ver Apéndice, Cuadro 1). Para el estudio de Validez de criterio respecto a la variable Intenciones de Elección de Carrera se diseñó un Cuestionario de Intenciones de Elección de Carrera (CIEC). El CIEC posee 54 ítems. Cada uno de ellos menciona una carrera de nivel superior y se solicita al individuo que indique en una escala de 1 a 10 sus probabilidades de elección de esa carrera. Todos los datos fueron ingresados y analizados en el paquete estadístico SPSS 9.0 (Manzano y et al., 1999).

\section{Construcción}

\section{Diseño y Análisis de Ítems}

En la etapa inicial de construcción del Inventario se elaboraron indicadores operacionales de cada uno de los constructos (inteligencias) de la teoría de Gardner $(1994 ; 1999)$ y de las asignaturas cursadas por los adolescentes de la muestra, relacionadas con cada una de las inteligencias (por ejemplo, actividades curriculares de Música para la Inteligencia Musical). 
Se redactaron de este modo 144 ítems (18 por escala) que fueron entregados a 8 jueces expertos en Orientación Vocacional, Psicología Educacional, Construcción de pruebas, Teoría de las Inteligencias Múltiples y Teoría de la Autoeficacia Percibida. Estos jueces evaluaron cada reactivo en función de criterios de claridad semántica, corrección sintáctica, adecuación al nivel educativo de los sujetos y congruencia con los constructos pertinentes de la teoría de Gardner (Nunnally y Bernstein, 1995; Thorndike, 1989).

El análisis de los expertos determinó que 48 reactivos fueran descartados. Se elaboró la prueba preliminar con un formato Lickert de 1 a 10 puntos. Se han demostrado las ventajas psicométricas que produce el uso de formatos con mas alternativas de respuesta ( 0 a 100 con intervalos de 10 puntos o 1 a 10) que el formato lickert tradicional y, además, se escogió una escala 1-10 por estar los adolescentes argentinos más habituados a esta escala de evaluación que a la de 0-100 (Pajares et al., 2001; Bandura, 2001).

Los 96 mejores reactivos de acuerdo al juicio de expertos fueron administrados a una muestra de 279 adolescentes (media de edad: 16,81; mujeres 50,6\%; varones 49,4\%). Se analizaron las correlaciones ítem-total de cada reactivo, distribución de frecuencia de las respuestas a cada reactivo y respuestas adicionales a preguntas formuladas a los adolescentes para evaluar la facilidad de comprensión de cada reactivo (Nunnally, 1995; Bandura, 2001). Las correlaciones ítem-total observadas fueron las siguientes: Lingüística, de .26 a .76; Lógico-Matemática, .32 a .82; Espacial, .18 a .81; Cinestésica, .25 a .83; Musical, .33 a .86; Interpersonal, .29 a 77; Intrapersonal, 28 a .76 y Naturalista, de .21 a .76 .

Los reactivos de menor correlación con el puntaje total de la escala (inferiores a .30), con correlaciones que no alcanzaran un nivel de significación de .01 o que resultaran ambiguos o poco conocidos para la muestra de investigación fueron eliminados (Nunnally y Bernstein, 1995; Kehoe, 1995). De este modo fueron retenidos 72 reactivos y se elaboró una segunda versión preliminar del Inventario.

Finalmente se aplicó Análisis Factorial a los reactivos de cada escala con la finalidad de verificar la unidimensionalidad de los mismos y la homogeneidad de cada escala (Bandura, 2001; Nunnally y Bernstein, 1995). La prueba revisada fue Evaluar. 2003, $n^{\circ} 3$ (julio) 
administrada a una muestra de 500 adolescentes (mujeres 57,4\%) con una media de edad de 16.92 años. Se empleó Análisis Factorial Exploratorio con método de extracción Máxima Probabilidad (Joreskog \& Lawley, 1968) y rotación Oblimin, que se cree es el procedimiento que produce la mejor estimación de parámetros (Pedhazur, 1982).

Los ítems de la escala Matemática (9 ítems), Cinestésica (9 ítems), Musical (9 ítems), Intrapersonal ( 8 ítems), Naturalista ( 9 ítems) y Espacial (9 ítems) configuraron, respectivamente, un solo factor. El análisis factorial reveló dos factores en los nueve ítems iniciales de la escala Lingüística. Retirando el reactivo 2 (Escribir poesía) que no cargaba apropiadamente en el primer factor se obtuvo un único factor con 8 ítems. Fue necesario quitar el ítem 49 (Asistir a personas con necesidades especiales), el de menor carga factorial, para obtener un solo factor en la escala Interpersonal ( 9 ítems finales).

Se replicó el análisis factorial con una muestra de 917 adolescentes (Mujeres 54.3\%; media de edad: 17.18). Los ítems de las escalas Linguística, Lógico-Matemática, Cinestésica-Corporal, Musical, Interpersonal, Intrapersonal y Naturalista configuraron un sólo factor, respectivamente. En la escala Espacial se obtuvieron dos factores.

Retirando el ítem 23 "Hacer diseño con computadora", el de menor carga factorial, se obtuvo un factor con 8 reactivos. De este modo, se pudieron obtener 69 reactivos unidimensionales que conforman el Inventario en su versión final.

\section{Confiabilidad}

La confiabilidad de una medida alude al grado a que la misma se encuentra libre de error y sus dos principales dimensiones son la consistencia interna, vale decir el grado en que los ítems de una prueba miden el mismo constructo, y la estabilidad, que refiere a que diversas medidas con un mismo instrumento arrojen resultados sin variación significativa (Tornimbeni, Pérez y Baldo, 2000).

\section{a) Consistencia Interna}

Con los ítems seleccionados mediante el Análisis Factorial en la muestra precedente $(\mathrm{n}=917)$ se estimó la consistencia interna de cada escala mediante el procedimiento de Cronbach. Este método se basa en el análisis de las intercorrelaciones promedios entre los reactivos a partir de una sola aplicación de la prueba (Thorndike, 
1989). Los coeficientes alfa obtenidos se presentan en la Tabla 1 y puede observarse que todos los valores sen elevados.

Tabla 1: Coeficiente Alfa de Cronbach para cada escala del inventario

\begin{tabular}{ll}
\hline \multicolumn{1}{c}{ Escalas } & $\alpha$ \\
\hline Intrapersonal & .86 \\
Naturalista & .91 \\
Lingüística & .85 \\
Matemática & .89 \\
Espacial & .91 \\
Cinestésica & .93 \\
Musical & .93 \\
Interpersonal & $\mathbf{. 8 5}$ \\
\hline
\end{tabular}

\section{b)Estabilidad}

La correlación entre dos aplicaciones de una misma prueba, separadas por un intervalo de tiempo, informa sobre la estabilidad de esa medida (Cronbach, 1998). Si una medida representa la condición relativamente perdurable de un rasgo debe permanecer estable durante el período en el que los puntajes fueron empleados para este propósito (Nunnaly \& Bernstein, 1995). Para evaluar la estabilidad del IAMI se empleó el procedimiento test-retest; la prueba fue administrada a una muestra de 119 sujetos (mujeres 55,6\%; varones 44,4\%; media de edad: 17.02) y, un mes después los mismos sujetos respondieron nuevamente al inventario. Los puntajes obtenidos en ambas aplicaciones fueron correlacionados ( $\mathrm{r}$ de Pearson) y se observan valores aceptables aunque moderados (Kline, 2000). Los coeficientes de correlación para cada escala se presentan en la tabla 2 . 
Tabla 2: Coeficiente de correlación (test-retest) de cada escala del inventario

\begin{tabular}{lc}
\hline \multicolumn{1}{c}{ Escalas } & r \\
\hline Intrapersonal & $\mathbf{. 7 0}$ \\
Naturalista & $\mathbf{. 7 4}$ \\
Lingüística & $\mathbf{. 7 1}$ \\
Matemática & $\mathbf{. 7 6}$ \\
Espacial & $\mathbf{. 7 0}$ \\
Cinestésica & $\mathbf{. 8 0}$ \\
Musical & $\mathbf{8 0}$ \\
Interpersonal & $\mathbf{. 8 2}$ \\
\hline
\end{tabular}

Validez

En un sentido general, un instrumento de medición es válido si cumple satisfactoriamente con el propósito para el que fue construido (Nunnally, 1991). Las nuevas Normas Técnicas de la APA (1999) presentan a la validez como un proceso unitario y continuo y sustentado por diferentes tipos de evidencias.

\section{a)Evidencia de la estructura interna del test}

Haber obtenido ítems unidimensionales mediante Análisis Factorial, como se describió en el apartado de análisis de ítems, permite esclarecer la naturaleza de los rasgos medidos por cada escala y es una evidencia de la estructura interna de la prueba (Thorndike, 1989; APA, 1999). Otro procedimiento importante es verificar la independencia de las escalas mediante sus intercorrelaciones observadas. Con los sujetos de la muestra precedente $(\mathrm{N}=917)$, se estimaron las intercorrelaciones entre las escalas y se observaron valores de bajos a moderados (en constructos relacionados teóricamente), lo cual demuestra la relativa independencia de las escalas. La Tabla 3 presenta los coeficientes de correlación entre las escalas. 
Tabla 3. Intercorrelaciones entre las escalas del inventario

\begin{tabular}{|c|c|c|c|c|c|c|c|c|}
\hline & Lingüística & Lógico-Mat. & Espac. & Cinestésica & Musical & Intrapers. & Interpers. & Naturalista \\
\hline Lingüística & - & .234 & .205 & .099 & .237 & .410 & .541 & 210 \\
\hline Lógico-Mat. & & - & .331 & .326 & .052 & .086 & .252 & .292 \\
\hline Espacial & & & - & .363 & .178 & .101 & .153 & .375 \\
\hline Cinestésica C. & & & & - & .167 & .126 & .246 & .366 \\
\hline Musical & & & & & - & .213 & .243 & .249 \\
\hline Intrapersonal & & & & & & - & .534 & .163 \\
\hline Interpersonal & & & & & & & - & .252 \\
\hline Naturalista & & & & & & & & 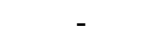 \\
\hline
\end{tabular}

\section{b)Evidencia de Diferencias entre Grupos Contrastados}

Si la teoría acerca del dominio medido postula que determinado grupos de la población poseen comparativamente niveles altos o bajos del dominio en cuestión, se puede aportar evidencia para la validez de la prueba comparando las puntuaciones obtenidas por esos grupos en el instrumento a validar (Thorndike, 1989). En un estudio efectuado para aportar este tipo de evidencia, se compararon los puntajes medios entre varones y mujeres, en cada una de las escalas del IAMI, utilizando prueba " $t$ " de diferencia entre medias ( $\mathrm{N}=917)$. Se observó que las mujeres superan significativamente a los varones en las escalas Lingüística, Musical, Intrapersonal e Intrapersonal y los varones, como grupo, obtienen puntuaciones medias más elevadas que las mujeres en las escalas Lógico-Matemática, Espacial y Cinestésica (superiores a .001). No se observaron diferencias significativas entre ambos sexos en la Escala Naturalista. Estos resultados son absolutamente congruentes con la investigación en diferencias entre sexos en habilidades objetivas y autoeficacia (Valiante, 2000).

\section{c)Evidencia de las relaciones test-criterio}

En este contexto la validez de una prueba es soportada por la efectividad con que se puede predecir, a partir de los resultados de la misma, el desempeño de una persona en una situación real diferente a la del test en sí (Tornimbeni, Pérez y Baldo, 2000). Los

Evaluar.

2003, $n^{\circ} 3$ (julio) 
instrumentos de medición de la autoeficacia han demostrado ser predictivos, en general, de índices de rendimiento académico y de elección de opciones (Lent, Brown y Hackett., 1994). Por consiguiente, se efectuaron dos investigaciones tendientes a examinar las relaciones entre las pruebas y dos criterios de este tipo.

\section{1-Rendimiento Académico}

En un primer estudio para obtener este tipo de evidencia se administró el Inventario a una muestra de 343 estudiantes secundarios (dos últimos años) de ambos sexos (mujeres 54.5\%) con una media de edad de 17,6 años. El Inventario se aplicó a comienzos del año lectivo y se registraron los promedios de las calificaciones obtenidas por estos estudiantes en 31 asignaturas cuatro meses después (primer promedio cuatrimestral). Se correlacionaron (coeficiente $r$ de Pearson) las puntuaciones totales de cada una de las 8 escalas versus todos los promedios de las asignaturas. La escala Lingüística se correlaciona significativamente (correlaciones superiores a .01) con el promedio de las asignaturas Formación Cívica (.29), Literatura (.27), Historia (.39), Filosofía (.30), Inglés (.25) y Psicología (35.). La escala Lógico-Matemática muestra correlaciones significativas (superiores a .01) con las asignaturas Filosofía (.26), Física (.28), Contabilidad (.33), Estadística (.57), Matemática (.21), Computación (.32) y Química (.18). La escala Espacial evidencia correlacionarse significativamente (>.01) con el promedio de las asignaturas Composición Gráfica (.31), Imagen y Composición Visual (.43), Historia del Arte (.34) y Dibujo Técnico (.38). La escala Corporal correlaciona significativamente (>.01) con el promedio de Educación Física (.32). La escala Musical correlaciona significativamente $(>.01)$ con el promedio de la asignatura Música (.26). La escala Interpersonal correlaciona significativamente (>.01) con el promedio de Psicología (.37) y Formación Cívica (.21). La escala Intrapersonal correlaciona significativamente con el promedio en Psicología (.28). Finalmente, la Escala Naturalista no se correlaciona significativamente con el promedio de los estudiantes en la asignatura Biología (.09), observándose correlaciones significativas (.32) con el promedio de Química de los Alimentos. 


\section{2-Intenciones de Elección de Carrera}

En otro estudio de validez predictiva se aplicó el Inventario a una muestra de 472 estudiantes secundarios de ambos sexos (mujeres 54.7\%) que cursaban el último año de su educación media y a fines del ciclo lectivo se les administró el Cuestionario de Intenciones de Elección de Carrera. Las puntuaciones de cada escala del Inventario fueron correlacionadas con todos las intenciones de elección de carrera del Cuestionario. Las correlaciones significativas $(>.01)$ y superiores a .20 entre cada escala

y las intenciones de elección de carrera asociadas fueron las siguientes. La escala Lingüística se correlaciona con la intención de elección en Letras (.34), Trabajo Social (.34), Filosofía (33), Psicología (.32), Ciencias de la Educación (.30), Idioma Extranjero (.27), Relaciones Internacionales (.21), Historia (.20), Comunicación Social (.20) y Relaciones Públicas (.20). La escala Lógico-Matemática correlaciona con Matemática (.53), Física (.51), Química (.43), Ciencias Económicas (.33), Computación (.28), Ingeniería Industrial (.27), Ingeniería Electrónica (.26), Ingeniería Civil (.22), Ingeniería Electricista (.22), Ingeniería Mecánica (.20) y Bioquímica (.20).

La escala Espacial con Arquitectura (.43), Diseño Gráfico (.43), Artes Plásticas (.43), Diseño Industrial (.40) e Ingeniería Civil (.28). La escala Corporal con Educación Física (.55), Fuerzas de Seguridad (.28), Ingeniería Electrónica (.28), Ingeniería Mecánica (.26), Ingeniería Aeronáutica (.23), Ingeniería Industrial (.22) e Ingeniería Electricista (.21). La escala Musical con Música (.56) y Cinematografía (.30). La escala Interpersonal con Trabajo Social (.37), Sociología (.32), Filosofía (.30), Relaciones Públicas (.29), Teatro (.26), Ciencia Política (.25), Abogacía (.20) y Relaciones Internacionales (.20). La escala Intrapersonal se correlaciona con las intenciones de elección de carrera en Psicología (.44), Psicopedagogía (.31), Filosofía (.25) y Teatro (.20). La escala Naturalista se asocia con las intenciones de elección de las carreras de Bromatología (.40), Agronomía (.35), Veterinaria (.34), Bioquímica (.31), Biología (.30), Odontología (.29), Enfermería (.28), Medicina (.25), Nutrición (.25), Farmacia (.23), Kinesiología (.22) y Geología (.20).

Evaluar. 2003, $n^{\circ} 3$ (julio) 


\section{Normas de Interpretación}

El Inventario fue administrado a una muestra de 936 estudiantes secundarios de los dos últimos años del nivel educativo polimodal con la finalidad de elaborar las Normas de Interpretación del mismo. Los Baremos son tablas de equivalencia entre puntuaciones directas y derivadas que permiten la comparación de los resultados individuales con los de un grupo de referencia (Grasso, 2000). En las medidas psicológicas cobran sentido puesto que las medidas psicológicas carecen de cero absoluto, no poseen unidades de medida constante y no son unívocas en ámbitos no especializados (Murat, 1985). La Transformación elegida en este caso fue la de Percentiles. Los percentiles son aquéllas transformaciones que se expresan en función del porcentaje de personas, en el grupo normativo, que quedan por debajo de una puntuación original determinada (Tornimbeni, Pérez \& Baldo, 2000). Se escogieron estas transformaciones no lineales por dos motivos: a) Son más fáciles de interpretar por un público no especializado y b) Es la transformación empleada por el Cuestionario de Intereses Profesionales (Fogliatto, 1991, 1993), instrumento al cual se integrará el IAMI en un Sistema de Orientación Vocacional Informatizado. Se construyeron Baremos diferenciados por sexo para cada escala puesto que ambos grupos de adolescentes (media de edad=17.14), varones $(\mathrm{N}=424)$ y mujeres $(\mathrm{N}=512)$, difieren significativamente en sus puntuaciones medias.

\section{Discusión}

Se ha desarrollado un Inventario de Autoeficacia en Inteligencias Múltiples (IAMI) dirigido a la evaluación de adolescentes de nuestro medio que desean ingresar a carreras de nivel superior. Cada una de las ocho escalas, relacionadas respectivamente con cada inteligencia del modelo de Gardner (1999), está compuesta por ítems unidimensionales que configuran un solo factor. Las escalas muestran correlaciones bajas a moderadas

entre sí (este último caso en escalas que representan constructos teóricamente relacionados, por ej. Lingüística vs. Interpersonal) lo que comprueba su relativa independencia y es una evidencia de validez de la estructura interna de las mismas.

La consistencia interna de cada escala fue verificada mediante el procedimiento alfa de Cronbach, observándose valores elevados para todas las escalas. La Estabilidad resulta adecuada para todas las escalas con valores más moderados que los obtenidos en consistencia interna. Los estudios de validez relacionada con criterios demuestran la 
utilidad predictiva de las escalas para ser empleadas en evaluación del comportamiento vocacional.

Todas las escalas correlacionan significativamente con criterios de rendimiento académico (promedio de calificaciones semestral) y de elección de carrera (intenciones expresadas de elección de carreras) congruentes, con valores más altos con relación a esta última variable. Estos resultados son consistentes con la literatura de autoeficacia en desarrollo de carrera, donde se ha demostrado extensamente que aquélla variable se correlaciona positivamente con diversos índices de éxito académico y elección de carrera (Lent; Brown, \& Hackett., 1994; Multon, Brown \& Lent, 1991).

El IAMI será integrado, en fases posteriores de investigación, al Sistema de Orientación Vocacional Informatizado, SOVI 2001 (Fogliatto \& Pérez, 2001). Esto facilitará a los orientadores de la región contar con un instrumento que les permita optimizar la evaluación que efectúan, puesto que en América Latina la Orientación Vocacional comprende, primordialmente, evaluación de intereses y suministro de información académica y ocupacional como estrategias de trabajo, descuidando la medición de otras variables críticas tales como autoeficacia, habilidades y rasgos de personalidad .

\section{Referencias}

Ameisen, J.C. (1996). The origin of programmed cell death. Science, 272: 1278-1279. American Psychological Association (APA) (1999). Standards for Psychological and Educational Tests. Washington, DC.

Aube, M. (1980). Amusia: Clinical and Computerized Scanning Correlations. Neurology. 30: 359-365.

Bandura, A. (1987). Pensamiento y acción. Barcelona: Martínez Roca.

Bandura, A. (1997). Self-efficacy: The exercise of control. New York: Freeman.

Bandura, A. (2001). Guía para la construcción de escalas de autoeficacia. Evaluar, II, 114-131. Universidad Nacional de Córdoba.

Bear, D.(1981). Hemispheric specialization and the Neurology of Emotion. Tesis Inédita. Harvard Medical School.

Evaluar.

2003, $n^{\circ} 3$ (julio) 
Blumer, D. (1975). Personality Changes with Frontal and Temporal Lobe Lesions. Psychiatric Aspects of Neurological Disease. New York: Grune \& Stratton.

Crombach, L (1998). Fundamentos de la Exploración Psicológica.. Madrid: Pirámide.

Fogliatto, H. (1991). Cuestionario de Intereses Profesionales. Manual. Buenos Aires: Guadalupe.

Fogliatto, H. (1993). Cuestionario de Intereses Profesionales Computarizado (CIPC). Manual y Diskette. Buenos Aires: Guadalupe.

Fogliatto, H. \& Pérez, E. (1997). Sistema de orientación vocacional informatizado (SOVI). Manual y diskette. Buenos Aires: Guadalupe.

Fogliatto, H. \& Pérez, E. (2001). SOVI 2001. Manual y CD-ROOM. Buenos Aires: Guadalupe

Gardner, H. (1975). The Shattered Mind. New York: Knopf.

Gardner, H. (1994). Estructura de la mente: Teoría de las inteligencias múltiples. México: Fondo de Cultura Económica.

Gardner, H. (1999). Intelligence reframed: Multiple intelligences for the 21st century. New York: Basic Books.

Gazzaniga, M. S. (1998). Groundbreaking work that began more than a quarter of a century ago has led to on going insights about brain organization and consciousness. Scientific American, 278: 51-55.

Gervais, A. (1982). Complex Mathfor a Complex Brain. Science News.

Geschwind, N. (1979). Specializations of the human brain. Scientific American, 241,180-1999.

Grasso, L. (2000). Introducción a la Estadística aplicada a las Ciencias Sociales y del Comportamiento. Universidad Nacional de Córdoba.

Hackett, G., \& Betz, N. (1981). A self-efficacy approach to the career development of women. Journal of Vocational Behavior, 18, 326-339.

Hackett, G., \& Lent, R. (1992). Theoretical advances and current inquiry in career psychology. In S. D. Brown \& R. Lent (Eds.). Handbook of counseling psychology. New York: Wiley.

Joreskow, K., \& Lawley, D. (1968). New Methods in maximum likelihood factor analysis. British Journal for Mathematical and Statistical Psychology, 21, 85-96. 
Kandel, E., Schwartz, J. \& Jessel, T. (1997). Neurociencia y conducta. Madrid: Prentice Hall.

Kehoe, J. (1995). Basic item analysis for multiple-choice tests. ERIC Digest. ED 398237.

Kline, P. (2000). Handbook of Psychological Tests. London: Rotledge e

Lent, R., Brown, S. \& Hackett, G. (1994). Toward a unifying social cognitive theory of career and academic interest: Choice and performance. Journal of Vocational Behavior, 45, 79-122.

Luria, A. (1966). Higher Cortical Functions in Man. New York: Basic Books.

Manzano, V., Varela, J., García, A., \& Pérez, F. (1999). SPSS para Windows. Madrid: Rama Editorial.

Multon, R., Brown, S., \& Lent, R. (1991). Relation of self-efficacy beliefs to academic outcomes: A meta-analytic investigation. Journal of Counseling Psychology, 38, 30- 38 .

Murat, F. (1985). Evaluación del comportamiento humano. Universidad Nacional de Córdoba.

Nauta, W. (1981). The Problem of the Frontal Lobe. A reinterpretation. Journal of Psychiatric Research. 8: 167-187.

Nunnally, J. (1991). Teoría psicométrica. México: Mac Graw Hill.

Nunnally, J. \& Bernstein, I. (1995). Teoría psicométrica. México: Mc Graw Hill.

Pajares, F. Hartley, \& Valiante, G. (2001). Response Format in Writing Self-Efficacy Assessment: Greater Discrimination Increases Prediction. Measurement and Evaluation in Counseling and Development. 33 (4), 214-221.

Pedhazur, J. (1982). Multiple regression in behavioral research: Explanation and prediction. New York: Harcourt Brace.

Roy, E. (1982). Action and Performance. Normality and Pathology in Cognitive Functions. Londres: Academic Press.

Shearer, B. (1996). The MIDAS. A Professional Manual. United States of America: Edición del Autor.

Evaluar.

2003, $n^{\circ} 3$ (julio) 
Shearer, B. (1999). The Application of Multiple Intelligences Theory to Career Counseling. Paper presented in the annual meeting of the American Educational Research Association. Montreal.

Thorndike, R. (1989). Psicometría aplicada. México: Editorial Limusa.

Tornimbeni, S., Pérez, E. \& Baldo, M. (eds) (2000). Introducción a los tests psicológicos. Córdoba: Brujas.

Valiante, G. (2000). Writing Self-Efficacy and Gender Orientation: A developmental Perspective. A Dissertation Proposal. Atlanta: Emory University.

Wasserstein, J. (1982). Evidence for diferentiation of right hemisphere visual perceptual functions. New York: New School for Social Research. 
Anexo

\section{CUADRO 1. INVENTARIO DE AUTOEFICACIA PARA INTELIGENCIAS MULTIPLES.}
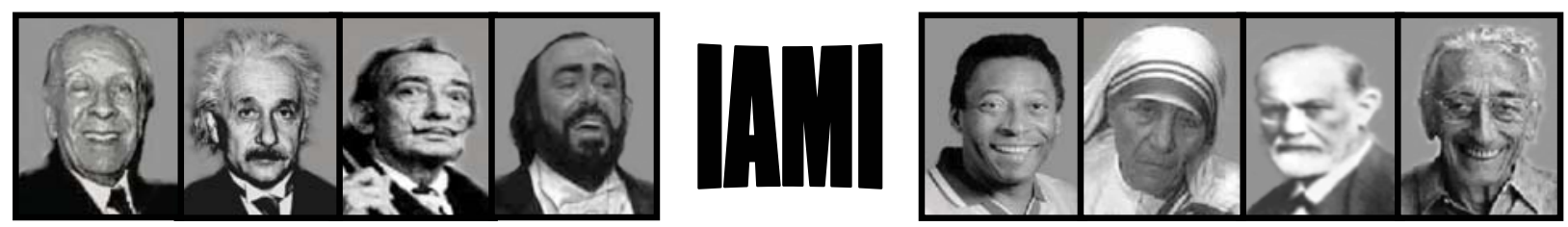

\section{Instrucciones}

El IAMI fue diseñado para ayudarte a planificar tus actividades de exploración de carreras. El IAMI comprende 69 ítems que se presentan en el Cuadernillo de Items y cada uno de ellos describe una actividad específica. Se te solicita que evalúes cuánta confianza posees en poder realizar exitosamente cada actividad.

Por favor emplea la siguiente escala para indicar tu nivel de confianza para desempeñar exitosamente cada una de las actividades mencionadas. Observa que la escala va de 1 (No puedo desempeñar esta actividad) a 10 (Estoy completamente seguro de poder desempeñar esta actividad exitosamente). Recuerda que puedes emplear cualquier valor entre 1 y 10 .

El siguiente ejemplo te ilustra el empleo de esta escala:

$$
\text { Item 00. Jugar Ajedrez }
$$

En el ejemplo, la persona ha escrito un 3 en el espacio a la derecha del ítem lo que indica que posee una escasa confianza en su habilidad para practicar ajedrez.

Ten presente que no se te pregunta sobre el agrado o desagrado que experimentas por las actividades mencionadas sino por la confianza que sientes en tu habilidad para desempeñarla exitosamente. Tampoco se solicita que evalúes la confianza que posees en tu habilidad potencial o futura, sino la confianza en tu habilidad actual para desarrollar exitosamente cada actividad. Contesta reflexivamente, no hay tiempo límite para completar el Inventario. Asegúrate de responder a todos los ítems y solicita ayuda al administrador del Inventario si no comprendes el significado de algún ítem.

Evaluar.

2003, $n^{\circ} 3$ (julio) 


\begin{tabular}{|c|c|}
\hline 1. Analizar obras literarias & \\
\hline 2. Crear composiciones literarias, tales como narrativa, poesía o textos dramáticos & \\
\hline 3. Reconocer géneros y estilos literarios & \\
\hline 4. Extraer las ideas principales de un texto & \\
\hline 5. Escribir con corrección gramatical & \\
\hline 6. Escribir textos periodísticos sobre temas de actualidad & \\
\hline 7. Redactar monografías o ensayos & \\
\hline 8. Expresarse con un vocabulario amplio y fluido & \\
\hline 9. Resolver problemas de la Física & \\
\hline 10. Obtener notas altas en Matemática & \\
\hline 11. Comprender estadísticas de encuestas o censos & \\
\hline 12. Resolver problemas geométricos & \\
\hline 13. Realizar operaciones matemáticas mentalmente & \\
\hline 14. Resolver ecuaciones de la Química & \\
\hline 15. Resolver problemas que requieran cálculos numéricos & \\
\hline 16. Utilizar calculadoras científicas & \\
\hline 17. Realizar tareas de contabilidad & \\
\hline 18. Dibujar motivos con precisión & \\
\hline 19. Dibujar objetos en tres dimensiones, tales como figuras geométricas & \\
\hline 20. Emplear la perspectiva en el dibujo & \\
\hline 21. Interpretar planos & \\
\hline 22. Diseñar construcciones & \\
\hline 23. Diseñar maquetas & \\
\hline 24. Realizar diseño gráfico & \\
\hline 25. Dibujar planos & \\
\hline 26. Tocar un instrumento musical como solista & \\
\hline 27. Leer partituras musicales & \\
\hline 28. Componer música & \\
\hline 29. Cantar armoniosamente junto a otras personas & \\
\hline 30. Cantar armoniosamente como solista & \\
\hline 31. Evaluar la afinación de un instrumento musical & \\
\hline 32. Tocar un instrumento en un grupo musical & \\
\hline 33. Escuchar una melodía sencilla y transcribirla en una partitura & \\
\hline 34. Realizar variaciones o arreglos de un tema musical & \\
\hline 35. Aconsejar a individuos con problemas personales & \\
\hline 36. Reconocer rápidamente los deseos e intenciones de otras personas & \\
\hline 37. Conducir un grupo de personas & \\
\hline 38. Exponer un tema en público & \\
\hline 39. Actuar en representaciones dramáticas, tales como rol play & \\
\hline 40. Defender los derechos de otras personas & \\
\hline 41. Entrevistarse con personas de mayor autoridad, tales como directivos escolares o empleadores & \\
\hline 42. Promocionar un producto o servicio & \\
\hline 43. Iniciar y mantener relaciones con diferentes personas & \\
\hline
\end{tabular}

Evaluar. 


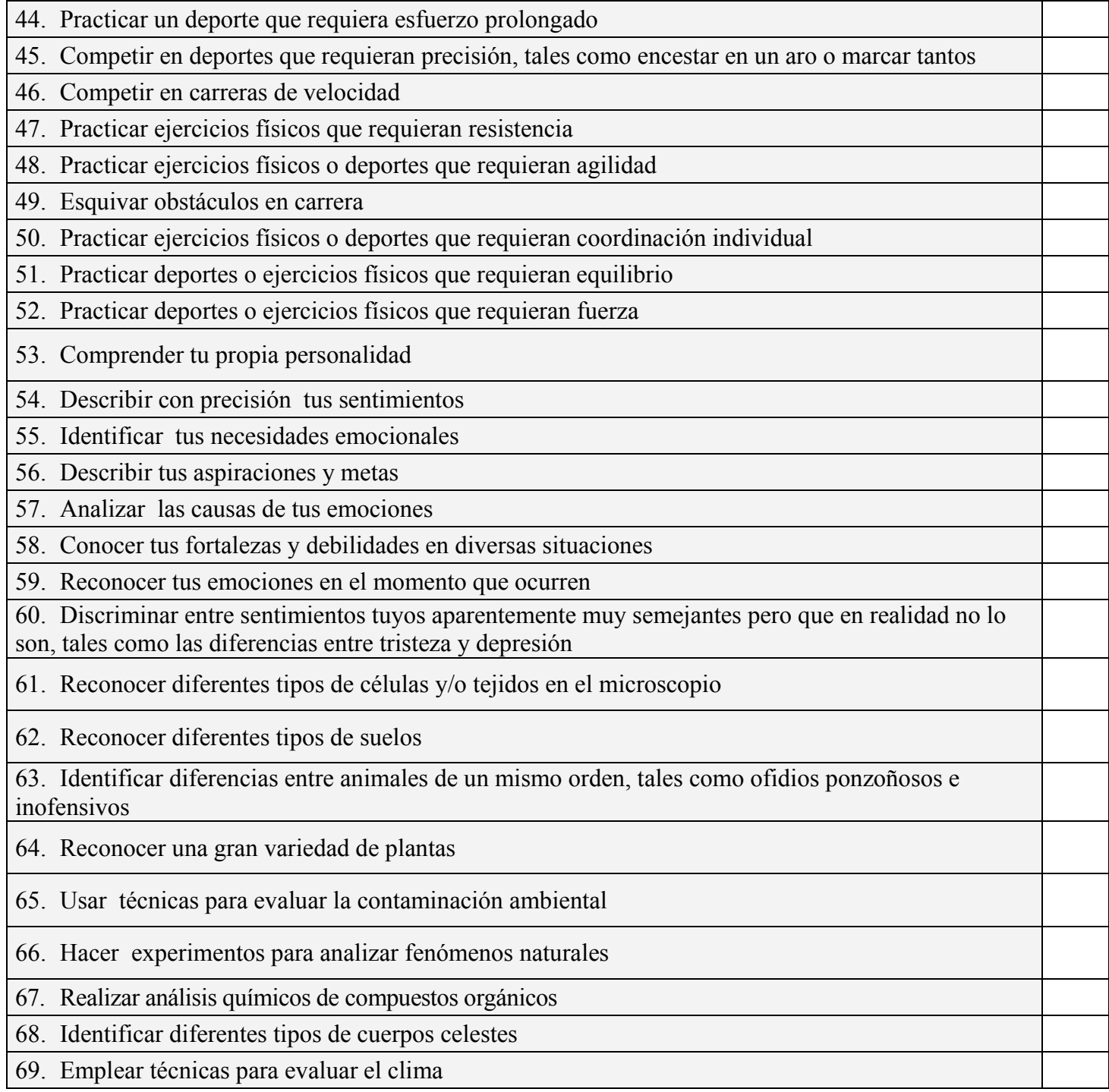

Evaluar.

2003, $n^{\circ} 3$ (julio) 
Figura 1- Esquema Representativo de las Lateralizaciones de la Función Cerebral.

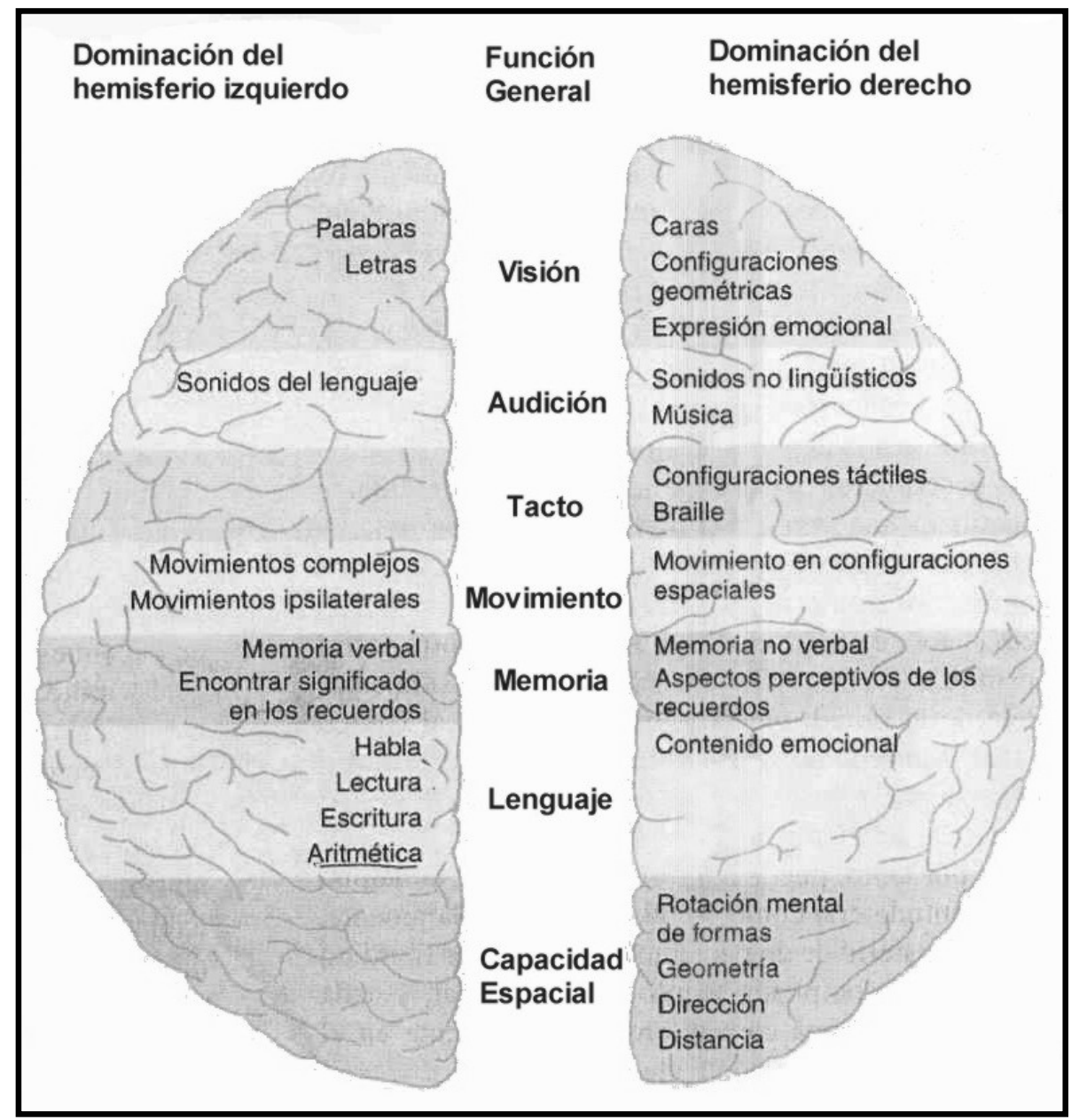

Evaluar.

2003, $n^{\circ} 3$ (julio) 
Figura 2 - Repetición de una palabra escrita de acuerdo con el modelo de Wernicke - Geschwind.

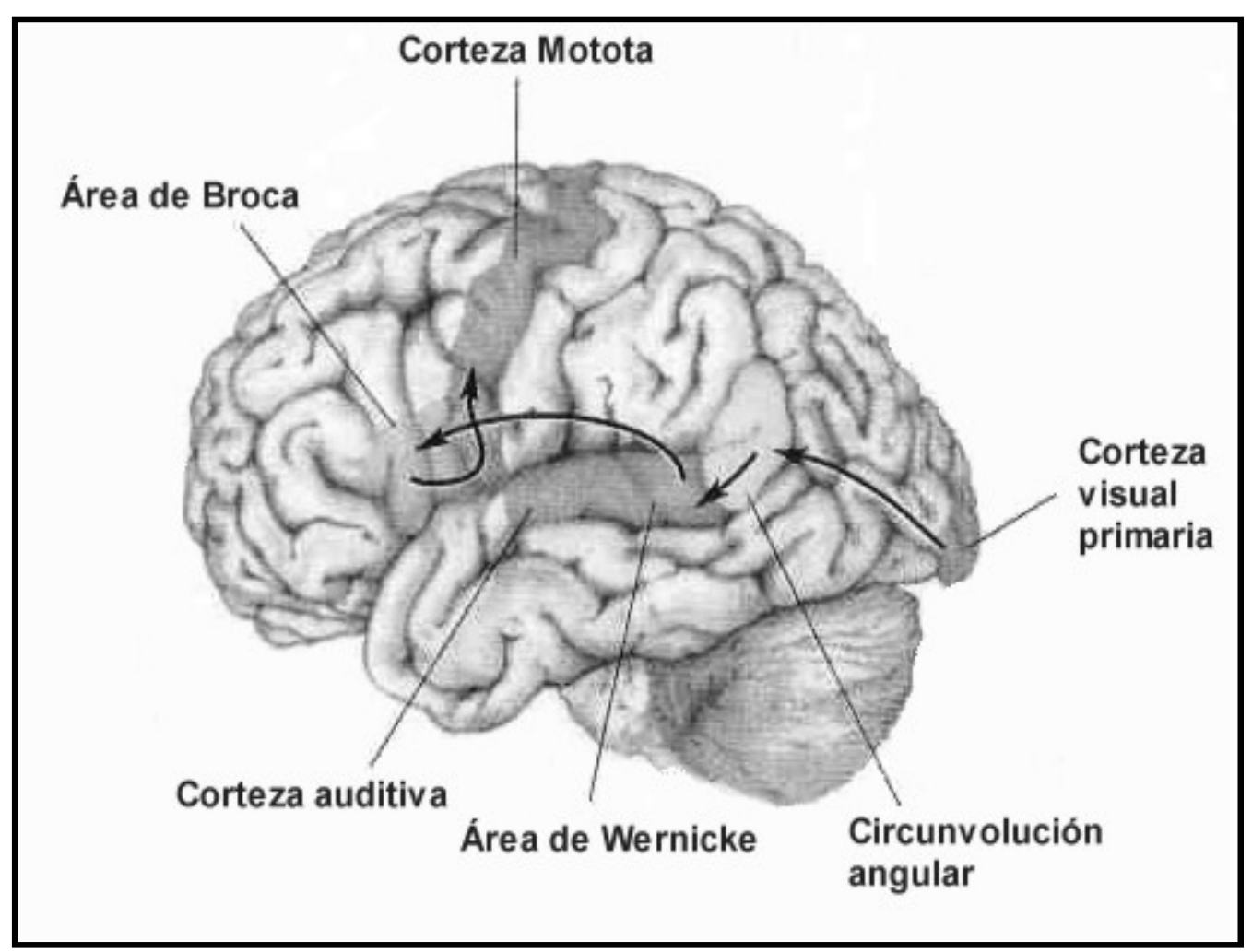


Figura 3 - Repetición de una palabra hablada de acuerdo con el modelo Wernicke-Geschwind.

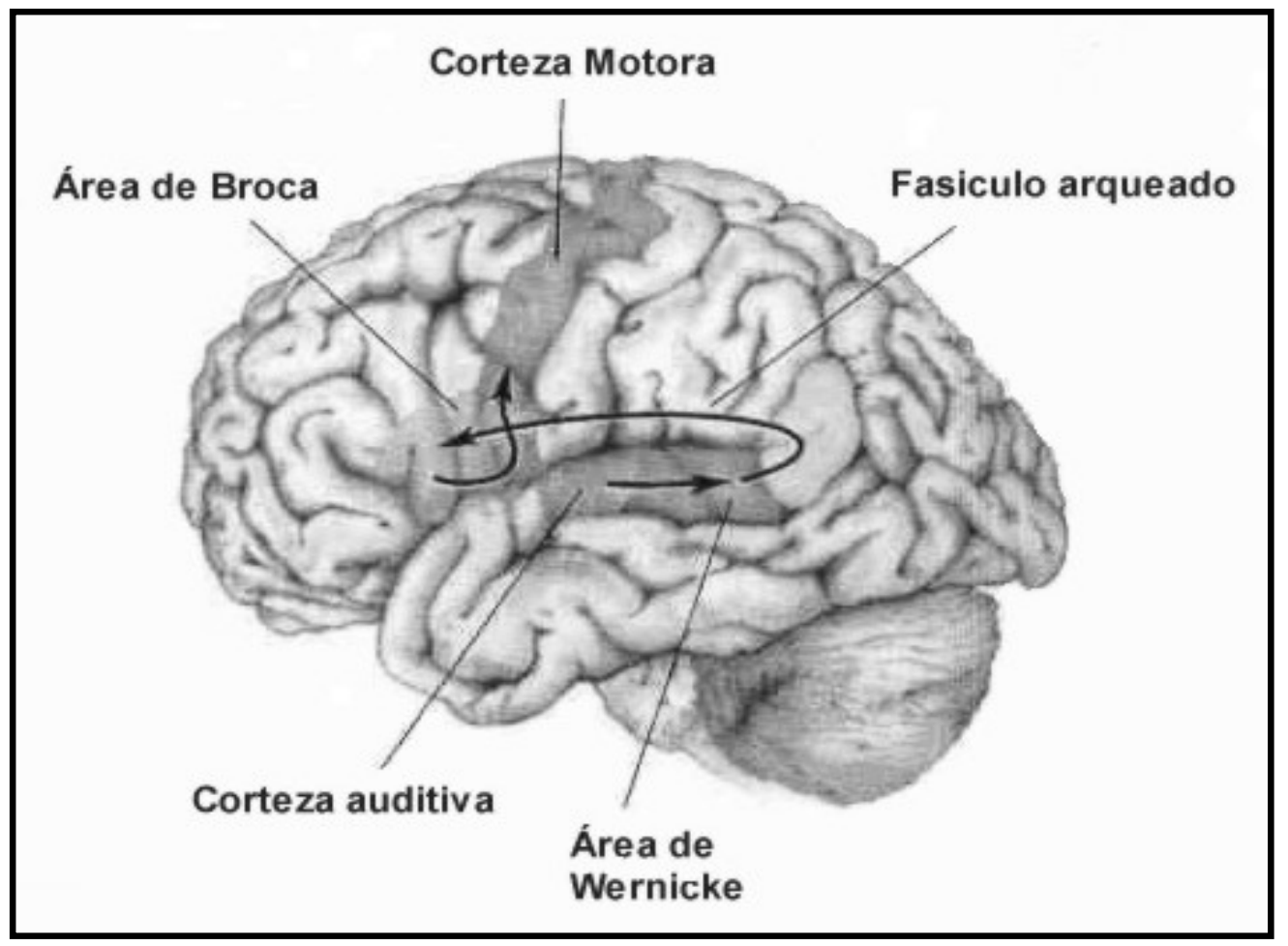

